\title{
Energy Efficient Clustering Using Fixed Sink Mobility for Wireless Sensor Networks
}

\author{
Muhammad Ali Khan \\ I.T Department, Hazara University, \\ Mansehra, Pakistan
}

Arif Iqbal Umar

I.T Department, Hazara University, Mansehra, Pakistan

\author{
Babar Nazir, \\ Department of CS, COMSATS Institute of IT, \\ Abbottabad, Pakistan
}

\author{
Noor ul Amin, \\ I.T Department, Hazara University, \\ Mansehra, Pakistan \\ Shaukat Mehmood, \\ I.T Department, Hazara University, \\ Mansehra, Pakistan \\ Kaleem Habib, \\ I.T Department, Hazara University, \\ Mansehra, Pakistan
}

\begin{abstract}
In this research an efficient data gathering scheme is presented using mobile sink as data collector with Clustering as sensor organizer in a randomly organized sensors in sensing field for wireless sensor network. The scheme not only extends the network lifetime through clustering process but also improves the data gathering mechanism through efficient and simplified mobile sink movement scheme. The cluster heads selection is based on both energy and data weight, which gathering all the data from the nodes within a cluster and then delivers it to the sink. The single mobile sink which visits the cluster heads as per defined path gathers data periodically. The scheme is organized through "Mobile Sink based Data Gathering Protocol (MSDGP)" which combines single message energy efficient clustering and data gathering process. For performance evaluation, the protocol is extensively simulated for different performance metrics, namely Residual energy consumption, Number of dead nodes during variable number of rounds and Network lifetime. The results proves that MSDGP is successful in achieving the defined objectives of energy efficiency and is capable of extending the network lifetime by increasing the number of rounds. Therefore, proposed protocol is more suitable for scenarios where sensor nodes generate variable amount of data.
\end{abstract}

\section{Keywords-Wireless Sensors Network; Mobile Sink; Clustering; Sensors}

\section{INTRODUCTION}

The current increase in the demand for usage of multimedia applications and their data in the field of Wireless Senor Network (WSN) anticipate an efficient infrastructure [1] for data gathering \& delivery mechanism as well as energy efficiency over clustered topology. Such energy efficient infrastructure can be achieved by reducing the number of hops for communications [2], [3]. Considerable research has been focused on developing a robust energy efficient navigation system for the sinks in WSNs. In the recent years, multiple approaches have been proposed for both clustering and sink mobility [4], which broadly falls into two categories of proactive and reactive approach. In proactive approach, the sensed data is stored on the specialized central nodes storage, which is later collected by the sink [5]. On the other hand, in reactive approach, the sensed data is collected directly from the sensing nodes by the mobile sink.

The added motivation behind this research work is the further study of different factors contributing towards the strategies concerning clustering process and mobile sink adaptation in WSN environment and to utilizing these factors to devise simpler and efficient mechanism to improve sensor network efficiency and performance involving energy consumption and network lifetime, and contribute to a better understanding for the feature research activities.

The recent growth in event based and user centric applications which target remote monitoring, surveillance, and other related applications will deeply benefits from this research. This research aims to target the most common issues faced during the clustering of sensors and movement of Mobile Sink [2] for data gathering within a sensor field to provided data gathering scheme with limited constraints.

This paper presents a fixed mobility based reactive protocol named "Mobile Sink based Data Gathering Protocol (MSDGP)". In this protocol, the sensor nodes with highest energy and highest amount of data are selected as cluster heads which gathers data from the normal nodes within the cluster. This data is stored unless the mobile sink (with predefined mobility) comes within the transmission range of the cluster heads and request for the aggregated data. Once the request is received, the cluster heads forward the data to the mobile sink.

To performance of the proposed protocol is evaluated for multiple parameters using intensive simulations. The results show the MSDGP incurs low energy consumption with less dead nodes over a period of time, extending network lifetime. The paper is further organized by presenting core related research readings in Section 2 and proposed research protocol 
in Section 3. The Section 4 details simulations, findings and results, whereas paper is concluded in Section 5.

\section{RELATED WORK}

In currently available literature, the researchers have concentrated on the different methodologies in this field of WSNs over the period of few decades both physically and in terms of their working. Enormous schemes have been designed to achieve optimum performance depending upon the purpose and their implementation. Architectural advancements have been done and two basic approaches [1] are mostly being followed; Static nodes with static sink / mobile sink and mobiles nodes with static sink / mobile sink, with experimentation with hybrid approaches as well.

Currently with the advancement in wireless technology, mobile sink or sink mobility is a hot topic and lot of work is being done in this field considering the large / multimedia type data targeting routing techniques for QoS and Network life time with goal of achieving efficient performance both in terms of routing and improved network life time (energy efficiency).

Sensors connectivity is utilized in schemes [5] to determine mobile sink path by locating data collection points at centroid of each cluster to minimizing multi-hop communications using travel salesman scheme for path determination. And schemes [2] where, MS path identification is based on message success rate from node to BS through information flooding over the network to find best path towards BS. Such schemes exert excessive inter-node communication and imbalanced energy usage due to direct communication with nodes.

Sink mobility techniques using dynamic virtually adjustable routing tree [3] using virtual backbone for routing data from CHs to edged circular mobile sink also limits energy efficiency as MS path consists of virtual tree and straight lines whereas cluster heads are selected nearest to the selected path. Each CH may follow multiple paths based on the location of MS causing overhead of path reconstruction. Whereas, direct mobile sink data gathering method based on centroid collection points in clusters [6] may collect data directly from all nodes but increase nodes-MS communication, limiting its implementation for large clusters.

Multiple specialized relays based data transfer schemes using pre-define movement of mobile sink, relays are selected based on point that $\mathrm{CHs}$ closest to mobile sink [7]. The constraint fixed trajectory of MS highlight the to-and-fro messaging neglects the energy consumption and multi hop data transfer rises data loss and collision. Similarly, [8] signal strength is considered for next-hop selection, whereas slow MS speed along its trajectory increases delay in data delivery to sink from high storage and energy abundance relays. In [9], protocol is applicable to limited scenarios, because the sink speed is fixed and the overall network lifetime is limited.

In [4], energy-hole problem is discussed through MS based Routing Protocol (MSRP) to increase life of network. CHs gather data from the normal nodes and wait until the sink arrives. On the arrival of the sink, the CHs send their data to sink. The sink considers energy of the $\mathrm{CHs}$ to schedule its data collection. Do to so, the sink records the energies information of CHs. As the sink gathers data from $\mathrm{CHs}$ and their neighbors to balance the energy consumption, this protocol provides enhanced network lifetime [10].

In [11], protocol called Virtual Circle Combined Straight Routing is proposed, using a combination of circles and straight lines to elect Cluster-Heads (CHs) and create a virtual backbone network. $\mathrm{CH}$ nodes collect data for delivering to sink using tree structure. The $\mathrm{CH}$ broadcasts request to sensors and form a tree to provide shortest path to the sink. The limitation of this protocol is that the sink follows only a circular path can cause longer delays in data collection. In [12], the authors proposed a protocol which visits subset of nodes to get sensed data. In this protocol, depositing sensed data at nodes and formation of virtual structure may cause an imbalance in energy and storage space. It is because the nodes and their next hops may quickly deplete their energy. Sensor nodes purposively has buffer space for sensed partial data storage, composed of data as well as compressed version of their neighbors. This protocol is suitable for scenarios where real time data is not a primary concern.

In [13], the authors propose a geographic routing protocol based scheme called Integrated Location Service and Routing ensuring MS packet delivery. MS, sensors, neighbor nodes all broadcast their location. MS movement is slow; network is static and MS hang on to at least one node on the network during one complete process. Target nodes near the sink are considered first for any changes in the data. The process of location update is time-stamped.

In [14], propose a protocol for delay-tolerant application. It focuses on self-aware MS for its future trajectories through broadcast of anticipated trajectories set to nodes. Relay nodes are used as mind points to route data to mobile sinks with trajectories information. Relays store all data for delivery to sink on its passing and predict other possible trajectories of the mobile sinks. This protocol provides improved data delivery, but incurs high energy consumption due to retransmissions. Similar concept [15] of mobile $\mathrm{CHs}$ which increase WSN lifetime for real time applications. The mobile $\mathrm{CHs}$ gathers data from the network before sending it to sink situated at the center of the sensing field. All moving CHs ensure connection with base-station during reporting. Three schemes are adapted for $\mathrm{CHs}$ movement to minimize communications and enhance network lifetime. The requirement of resource-rich mobile $\mathrm{CHs}$ is the major limitation of this scheme.

In [16], hierarchical cluster based architecture with large number of nodes, their CHs and one mobile sink is proposed. A predefined movement strategy through finding an optimum Hamiltonian Round calculated by sink according to Euclidean distance table provided by sink. Sink broadcasts message for $\mathrm{CHs}$ location which is replied with Cartesian coordinates, the sink calculates Euclidean distance between each $\mathrm{CH}$ and other $\mathrm{CHs}$ and between each $\mathrm{CH}$ and itself. In [17], scheme requires a single mobile sink for data collection. Do to so, the MS moves in a straight direction for data collection from nodes. This protocol focuses to minimize communications through efficient path planning with an ultimate goal of energy efficiency. To do so, a message is sent to the sink from where 
it is transmitted to the nodes and their neighbors. The sensor nodes report data to the sink when event of the similar interest occurs [18]. Moreover, acknowledgment is used to confirm successful packet delivery for each packet. The drawback is the limitation in fixed line path of mobile sink and avoiding delay.

The clustering protocol in [10], basis cluster heads selection on residual energy and centroid positioning of the node. Due to static sink, inter-cluster communication is done by utilizing cluster's overlapping nodes as anchor nodes or guard nodes. Excessive intra and inter cluster communication is done for data gathering.

The DEMC protocol is used to check the performance of MSDGP, and to evaluate if the selection of cluster heads on the basis of residual energy and data volume provides improved network lifetime then scheme of residual energy and the centroid positioning of that specific node.

\section{THE PROPOSED PROTOCOL}

A WSN consists of a set of nodes $\mathrm{V}$, where $\mathrm{v}$ represents a single node and $v \in V$. Each node is recognized by a unique identifier, whereas all nodes communicate via full-duplex links. Moreover, a cluster is a subgroup of $\mathrm{V}$, and multiple clusters cover the entire network.

The assumptions for our network model are listed as follows:

- Randomly deployed sensor in the sensing field.

- Nodes will be stationary.

- Nodes will use fixed transmission power.

- The sink is mobile with rich resources.

- Nodes unaware of their location information.

MSDGP uses three types of nodes, mentioned as follows:

1) Normal nodes: Nodes that sense information from the surroundings.

2) Cluster-heads: The nodes accountable to collect data from the normal nodes.

3) Sink node: The node that collects data from the clusterheads and provide it to the outer world.

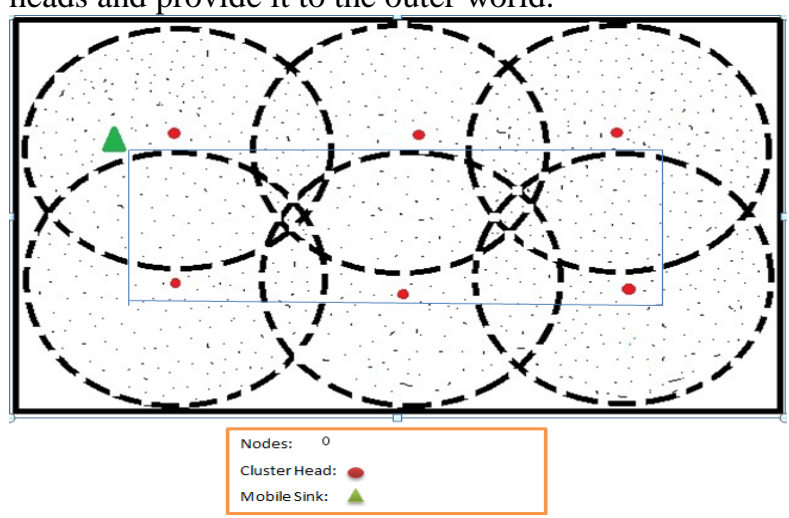

Fig. 1. General Structure of proposed WSN

\section{B. MSDGP Operation}

All the sensors are randomly deployed in the target sensing field; clustering is performed where cluster heads are selected through election between sensors on the basis of both data stored and residual energy of involved sensors. Once the cluster heads are selected, the normal nodes associate themselves with the cluster heads and forward their sensed readings to that cluster heads within a cluster. Once the data is gathered, cluster heads forward their collected sensed readings to specialized mobile sink whenever it comes within the vicinity or in their transmission range. The proposed is a fixed mobility based reactive wireless sensor networks with basic objective behind the selection of cluster heads on the basis of energy and data is to minimize the overall intra cluster communication to achieve extended network lifetime.

\section{MSDGP cluster formation}

The formation of the clusters for the sensor nodes is purely based on selection of their cluster heads. Therefore, cluster heads must be selected in an optimal way. MSDGP selects the cluster heads by calculating residual energy (E) and size of the sensed data (D) of the sensor nodes. To do so, each node calculates a weight (W) considering $\mathrm{E}$ and $\mathrm{D}$ and makes a decision of becoming a cluster head. The weight can be calculated as:

Where,

$$
\mathrm{W}=(\alpha \times E)+(\beta \times D)+(\mu \times I)
$$

$$
0<\mu<\beta<\alpha<1 \text {. }
$$

The weight $(\mathrm{W})$ is a linear combination of $\mathrm{E}, \mathrm{D}$, and I, where $\mathrm{E}$ has greater proportion in weight calculation followed by D and I, respectively. It is because, we have given energy the highest importance followed by size of the sensed data, whereas I works as a tie breaker incase two nodes have similar energy and data size. Once, every node calculates its weight, a timer (T) (delay) is set for the broadcast of a clustering request message as per the following:

$$
T=\frac{1}{W}
$$

In the initialization phase, all nodes assume that they are the cluster-heads, therefore, they set their cluster-head flag to true. However, when the respective timers of the nodes are reached, the nodes broadcast their cluster message as per weight of the node. When the other nodes receive the clustering message, they simply compare their received weight to their local weight. If the received weight is greater, they set the node (from which the message is received) as their clusterhead. Moreover, then mark self-cluster head flag to false, cancelling scheduled clustering request.

\section{MSDHP Intra-cluster communication}

Once the clustering is performed and completed, the remaining of the nodes associate themselves with the cluster heads and start reporting their sensed data to that respective cluster heads based on saved cluster-head ID. The cluster head on receiving the data performs aggregation and sends that data to available mobile sink, normally when cluster head is within the transmission range to that mobile sink. 


\section{E. Mobile Sink Data Collection}

In MSDGP, the sink follows rectangular mobility model and covers the entire network crossing the clusters formed. It sends data requests to cluster heads within the transmission range and collects the aggregated data. In this way, the sink collects the aggregated data from the cluster-heads. The data gathering phase depends on the speed of the mobile sink and is long enough in which a mobile sink can traverse throughout the network.

\section{F. MSDGP Algorithm}

The following sub section presents the algorithm being employed for MSDGP, It has been divided into four phased namely Initialization phase, Phase1, Phase2, and Phase3. The initial phase is responsible for information gathering required for initial setup and working of the preceding phases. In phase 1 , clustering is done, which includes selection of cluster head. Phase 2 carries out data gathering by $\mathrm{CH}$ from normal nodes. Phase 3 is responsible for gathering data from $\mathrm{CH}$ through movement of mobile sink.

\section{Initialization phase:}

1. Deploy_Nodes()

2. $\quad I \leftarrow$ Assign_Node_ID()

3. $\quad E \leftarrow$ Assign_Default_Energy()

4. $\quad D \leftarrow$ Sense_Data()

5. $W=(\alpha \times E)+(\beta \times D)+(\mu \times I)$

6. $T=\frac{1}{W}$

7. Is_Cluster_Head $=$ True

8. Schedule_CH_Message $(T)$

\section{Phase 1:}

9. IF Timer_Expires()

10. Broadcast_CH_Message()

11. END IF

12. IF $C H \_M e s s a g e \_R e c e i v e d()$

13. IF Rec_W $>W$

14. Set_CH(Rec_Node_ID)

15. Is_Cluster_Head $=$ False

16. END IF

17. END IF

Phase 2:
18.
19.
20.

$$
\begin{aligned}
& \text { IF Normal_Node() } \\
& \text { Send_Data }\left(D, C H \_I D\right) \\
& \text { END IF }
\end{aligned}
$$

\section{Phase 3:}

$\begin{array}{lc}21 . & \text { IF Node_is_Sink() } \\ 22 . & \text { Broadcast_Data_Request() } \\ 23 . & \text { END IF } \\ 24 . & \text { IF Data_Request_Received }() \\ 25 . & \text { IF Node_is_CH() } \\ 26 . & \text { Send_Data }(D, \text { Sink_ID) } \\ 27 . & \boldsymbol{E N D} \boldsymbol{I F} \\ 28 . & \boldsymbol{E N D} \boldsymbol{I F}\end{array}$

\section{RESULTS AND DISCUSSION}

The comprehensive simulations have been organized to evaluate the performance of MSDGP using OMNET++, a simulator that uses INET [19] framework. INET framework consists of simulation modules that are specially designed for wireless sensor networks. The energy model that is presented in [20] is used based on which, the energy consumed in transmitting a $\mathrm{k}$ bit message over a distance $\mathrm{d}$ is calculated as:

$$
\begin{gathered}
E_{T x}(k, d)=E_{T x}-\operatorname{elec}(k)+E_{T x}-\operatorname{amp}(k, d) \\
E_{T x}(k, d)=E_{\text {elec }} \times k+E_{\text {amp }} \times k \times d^{2}
\end{gathered}
$$

Similarly, the energy consumed in receiving the message is calculated as:

$$
\begin{gathered}
E_{R x}(k)=E_{R x}-\operatorname{elec}(k) \\
E_{R x}(k)=E_{\text {elec }} \times k
\end{gathered}
$$

Where, $E_{T x}(k, d)$ represents, amount of energy that is needed to send $\mathrm{k}$ bit message over distance $\mathrm{d}$ meters. Similarly, $E_{R x}(k)$ represents the amount of energy that is needed to receive $\mathrm{k}$ bit message. Moreover, $E_{\text {elec }}$ and $E_{\text {amp }}$ represents the amount of energy required for using the transceivers and amplifier, respectively.

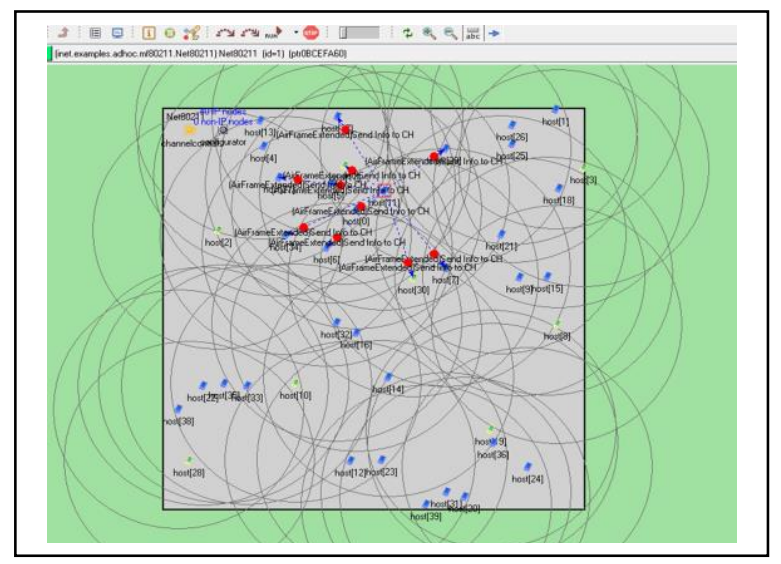

Fig. 2. MSDGP simulation using OMNET++

The simulation setup and other parameters for the simulation are detailed in table 1. All the protocols evaluated during the performance comparison have been setup using these parameters.

TABLE I. SimULATION SETUP

\begin{tabular}{|l|l|l|}
\hline Type & Parameter & Value \\
\hline \multirow{5}{*}{ Network } & Field dimensions & $1000 \times 1000$ \\
& Nodes location & Random \\
& Sink mobility model & Rectangular \\
& Sink initial energy & $9 \mathrm{~J} /$ battery \\
& Sink speed & Fixed $5(0-20 \mathrm{~m} / \mathrm{s})$ \\
& Nodes initial energy & $3 \mathrm{~J} / \mathrm{battery}$ \\
\hline \multirow{5}{*}{ Application } & Data packet size & Variable \\
& Broadcast packet size & 25 bytes \\
& Packet header size & 25 bytes \\
\hline
\end{tabular}




\begin{tabular}{|l|l|l|}
\hline & $E_{\text {elec }}$ & $50 \mathrm{~nJ} / \mathrm{bit}$ \\
& $E_{\text {amp }}$ & $0.0012 \mathrm{pJ} / \mathrm{bit} / \mathrm{m}^{4}$ \\
\hline
\end{tabular}

\section{A. Sum of Residual Energy:}

Figure 3, shows the results for the Sum of residual energy consumed by all the sensor nodes with respect to variable number of rounds. As MSDGP and MSDGP-E are designed for static sensor network with mobile sink, the overall energy consumption is recorded to be low as compared to DEMC as DEMC is designed for static sink or base station. However, during clustering phase both MSDGP and MSDGP-E uses single message clustering approach which plays important role in saving energy required for clustering as compared to DEMC which not only uses multi-messaging during clustering but also uses multi hoping for data delivery to base station which is avoided by both MSDGP and MSDGP-E. Considerable amount of energy is saved in both phases. The result shows that for all three schemes the energy consumption spikes during the middle rounds and then slowdowns due to the low concentration of the nodes towards the end.

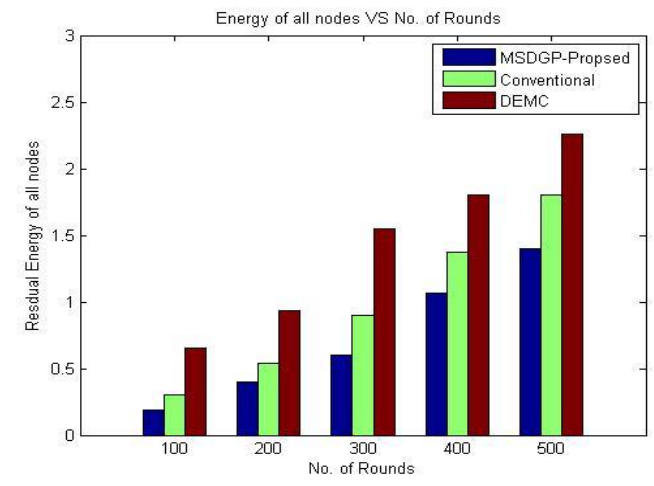

Fig. 3. Sum of residual energy of all nodes

\section{B. Number of Dead Nodes:}

Figure 4, shows the number of dead nodes with respect to variable number of rounds of the MSDGP, MSDGP-E and DEMC protocols. As projected in result and due to the fact that both MSDGP and MSDGP-E are based on similar sink mobility approach so there is only small difference among

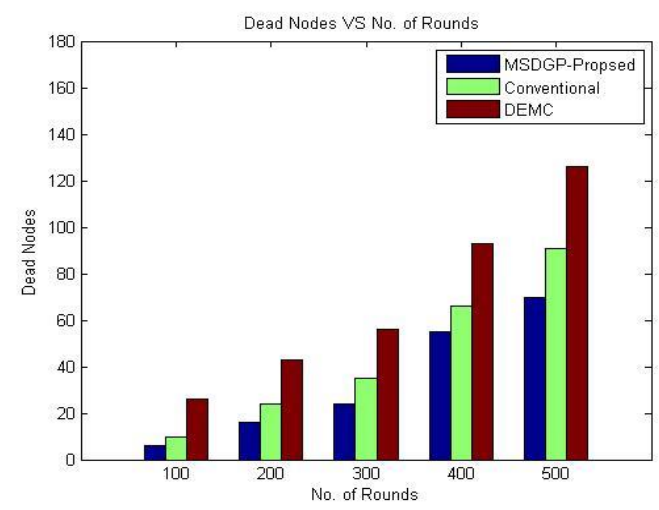

Fig. 4. Number of dead nodes w.r.t rounds their number of dead nodes due to clustering technique difference whereas DEMC being totally different approach shows major difference in number of dead nodes specially towards the end. The results proves that multi-hoping consumes more energy during inter-cluster communication.

MSDGP uses intelligent clustering mechanism that uses a single message for cluster formation. In addition, the clusterhead communicates with the sink only. Hence, it can be concluded that it also uses optimum number of clustering messages to achieve the required level of packet delivery ratio, which does not exceed one. However, under high mobility of the mobile sink, the packet loss may increases and the packet delivery ratio is affected.

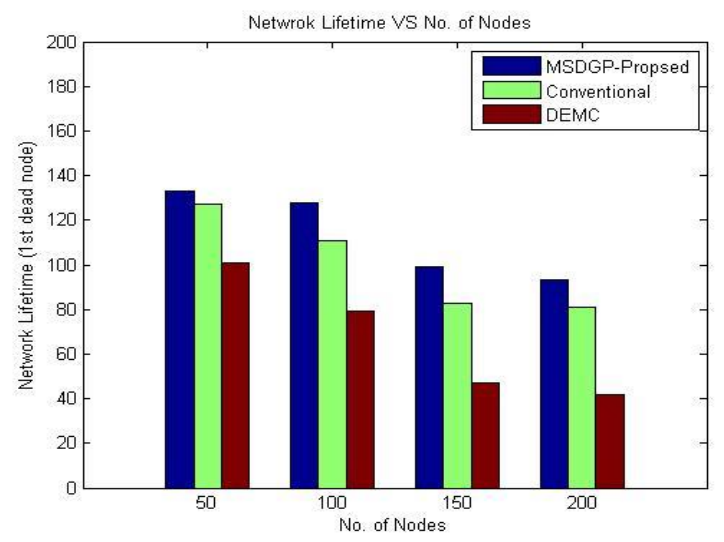

Fig. 5. Network lifetime w.r.t variable nodes

\section{Network Lifetime:}

Figure 5, presents the network lifetime of proposed MSDGP, conventional approach MSDGP-E and base approach of DEMC with respect to variable number of nodes being introduces into the system. As all three schemes uses same fixed transmission power as per the simulation setup, the overall network lifetime is shown as it fluctuate when the sensor nodes increases. Due to the fact that all three schemes select cluster heads using different approaches which make remarkable difference in their overall network lifetime. It is because; when concentration of nodes is less, lesser is the intra-cluster communication w.r.t clustering whereas selection of cluster heads in MSDGP (residual energy and data volume) reduces the overall communications in the network. Consequently, MSDGP provides improved energy efficiency (due to less communications) and extends the network lifetime as compared to DEMC where both intra and inter- cluster communication are being done with additional usage normal nodes as anchor nodes, which also do imbalanced energy consumption .

\section{CONCLUSION}

This paper presents a novel scheme for wireless sensor networks. It is a fixed mobility based reactive protocol using clustering based on the amount of sensed data and residual energy. The motive behind this cluster formation was to extend network lifetime by reducing the intra-cluster and eliminating inter-cluster communications. Results show that 
MSDGP achieved less energy consumption and provided extended network lifetime through implementation of single message $\mathrm{CH}$ selection process and by introduction of mobile sink instead of static sink.

\section{FUTURE WORK}

As a future work, QoS operations and data recovery mechanism are to be implemented into the proposed scheme, and compare it with more protocols and evaluate its performance using new performance metrics involving QoS parameters like throughput, fault tolerance, coverage areas and will also introduction hybrid approach by combing nodes mobility and more fixed Sinks.

\section{REFERENCES}

[1] Khan AW, Abdullah AH, Anisi MH, Bangash JI. A Comprehensive Study of Data Collection Schemes Using Mobile Sinks in Wireless Sensor Networks. Sensors (Basel, Switzerland). 2014;14(2):2510-2548. doi:10.3390/s140202510.

[2] Hyunjo Lee; Miyoung Jang; Jae-Woo Chang;," A New Energy-Efficient Cluster-Based Routing Protocol Using a Representative Path in Wireless Sensor Networks", International Journal of Distributed Sensor Networks Volume 2014, Article ID 527928, 12 pages.

[3] Chen, T.S.; Tsai, H.W.; Chang, Y.H.; Chen, T.C. 2013. Geographic converge cast using mobile sink in wireless sensor networks. Comput. Commun. 2013, 36, 445-458.

[4] Nazir, B.; Hasbullah, H. 2010. Mobile Sink Based Routing Protocol (MSRP) for Prolonging Network Lifetime in Clustered Wireless Sensor Network. In Proceedings of the IEEE International Conference on Computer Applications and Industrial Electronics (ICCAIE), Kuala Lumpur, Malaysia, 5-8 December 2010; pp. 624-629.

[5] Alhasanat, A.I., Matrouk, K.D., Alasha'ary, H.A. and Al-Qadi, Z.A. (2014) Connectivity-Based Data Gathering with Path-Constrained Mobile Sink in Wireless Sensor Networks. Wireless Sensor Network, 6, 118-128.

[6] Ilkyu H.; Mamurjon D.; Byoungchul A. "An Energy-Efficient Data Collection Method for Wireless Multimedia Sensor Networks", International Journal of Distributed Sensor Networks, Volume 2014 (2014), Article ID 698452, 8 pages.

[7] Zhongyu Zhang; Zunwen He; Jianguang Jia; Cunxiang Chen, "A new data gathering scheme for trajectory constrained mobile sink in WSN," Wireless Communications \& Signal Processing (WCSP), 2012 International Conference on , vol., no., pp.1,6, 25-27 Oct. 2012.

[8] Edgar H, and Callaway J. 2003. Wireless Sensor Networks: Architectures and Protocols: a CRC press company, Auerbach publications.
[9] P. Madhumathy; D. Sivakumar;," Reliable Data Gathering by Mobile Sink for Wireless Sensor Networks", International Conference on Communication and Signal Processing, IEEE April 3-5, 2014, 978-14799-3358-7114.

[10] S.Ali,S.Madani "Distributed Efficient Multi Hop Clustering Protocol for Mobile Sensor Networks", The International Arab Journal of Information Technology 8, no. 3, 2011.

[11] Kotsilieris, T.C.; Karetsos, G.T. 2013. "Prolonging the lifetime of twotiered wireless sensor networks with mobile relays". ISRN Sens. Netw. 2013, doi: 10.1155/2013/610796.

[12] Shi, L.; Zhang, B.; Mouftah, H.T.; Ma, J., 2013, DDRP: An efficient data-driven routing protocol for wireless sensor networks with mobile sinks. Int. J. Commun. Syst. 2013, 26, 1341-1355.

[13] Tang, B.; Wang, J.; Geng, X.; Zheng, Y.; Kim, J.U. 2012. "A novel data retrieving mechanism in wireless sensor networks with path-limited mobile sink". Int. J. Grid Distrib. Comput. vol.5, pp.133-140

[14] Aioffi, W.M.; Valle, C.A.; Mateus, G.R.; da Cunha, A.S. "Balancing message delivery latency and network lifetime through an integrated model for clustering and routing in wireless sensor networks". Comput. Netw. 2011, 55, 2803-2820.

[15] Jin Wang; Xiaoqin Yang; Zhongqi Zhang; Liwu Zuo; Jeong-Uk Kim, "Energy Efficient Routing Algorithm for Wireless Sensor Networks Supporting Mobile Sinks ", Advanced Science and Technology Letters Vol.49 (SoftTech 2014), pp.262-268

[16] Tacconi, D.; Miorandi, D.; Carreras, I.; Chiti, F.; Fantacci, R. 2010, Using wireless sensor networks to support intelligent transportation systems. Ad Hoc Netw. 2010, 8, 462-473.

[17] Tian, K.; Zhang, B.; Huang, K.; Ma, J., 2010. Data Gathering Protocols for Wireless Sensor Networks with Mobile Sinks. Proceedings of the IEEE Global Telecommunications Conference (GLOBECOM 2010), pp. $1-6$.

[18] Banerjee, T.; Xie, B.; Jun, J.H.; Agrawal, D.P. 2010. Increasing lifetime of wireless sensor networks using controllable mobile cluster heads. Wirel. Commun. Mob. Comput. 2010, 10, 313-336.

[19] Drytkiewicz W; Sroka S; Handziski W; Köpke H; Karl H; ” A Mobility Framework for OMNeT++"

[20] Witold Drytkiewicz, Steffen Sroka, Vlado Handziski, Andreas Köpke, and Holger Karl. Mobilty framework website. http://www-tkn.ee.tuberlin.de/research/ mobility-omnetpp-sim.

[21] Debroy Bijan Kumar, Sheikh Sadi and Md. Al Imran, "An Efficient Approach to Select Cluster Head in Wireless Sensor Networks", Journal of Communication, Vol. 6, No. 7, (October 2011).

[22] A. Kansal, A.A. Somasundara, D.D. Jea, M.B. Srivastava, and D. Estrin. "Intelligent fluid infrastructure for embedded networks".Proceedings of the 2nd International Conference on Mobile Systems, Applications, and Services (MobiSys'04), Boston, MA, USA, pp. 111-124, 2004. 